\title{
UMA TÉCNICA BASEADA EM IMAGENS PARA CORREÇÃO DA POSTURA DE PACIENTES NA AQUISIÇÃO DE TERMOGRAFIAS
}

An image-based technique for correction of the posture

of patients during the acquisition of thermographic images

\section{Denival Araujo dos Santos'; Leandro Augusto Frata Fernandes ${ }^{2}$}

Resumo Objetivos: UEste trabalho apresenta uma técnica desenvolvida para auxiliar no processo de captura de imagens térmicas de mama, na posição de captura T2 modificada, para diagnóstico de câncer de mama. Materiais e métodos: No desenvolvimento da técnica contamos com 49 termografias adquiridas na posição de captura T2 do banco de dados da Universidade Federal Fluminense. Destas, 32 foram capturadas sob a utilização de protocolo de captura estático e 17 de dinâmico com ventilação e álcool. Resultados: Para validação dos resultados, utilizamos um software de segmentação de mama, desenvolvido pela UFF, que considera como postura correta quando se consegue extrair a ROI. Em 42 imagens $(85,71 \%)$ conseguimos resultados iguais entre as duas abordagens, com diferença em apenas 7 imagens (14,29\%). Conclusão: A técnica mostrou-se robusta na verificação, para correção, da postura de pacientes que são submetidos a exames de termografia, o que possibilita, a partir da postura correta, um melhor imageamento das estruturas da mama que sejam úteis para diagnósticos médicos mais precisos, além de auxiliar métodos de diagnóstico assistido por computador.

Palavras-chave: Posicionamento, imagens térmicas, extração de ROI

Abstract Aims: This paper presents a technique developed to assist in the thermal imaging breast procedure in the Capture T2 position modified for diagnosis of breast cancer. Materials and Methods: In the development of the technique we rely on acquired 49 thermography capture T2 database Universidade Federal Fluminense position. Of these 32 were captured using static protocol capture and ventilation with 17 dynamic and alcohol. Results: To validate the results we use a software segmentation breast UFF developed by considering how correct posture when you can extract the ROI. In 42 images (85.71\%) got similar results between the two approaches are different in only 7 images (14.29\%). Conclusion: The technique proved to be robust in check, correction of the posture of patients who are submitted to thermography exams, enabling the correct posture from a better imaging of the breast structures that are useful for more accurate medical diagnoses, and auxiliary methods of computer-aided diagnosis.

Keywords: Posture, thermal imaging, ROI extraction 
Os avanços de técnicas de processamento de imagens que visam a segmentação e registro têm facilitado a extração e estudo de estruturas anatômicas e fisiológicas em imagens médicas. Essas técnicas se tornaram um componente fundamental em um grande número de aplicações, tais como monitoramento do crescimento de tumores e acompanhamento clínico.

Imagens utilizadas para fins médicos podem ser adquiridas por fontes diversas. São exemplos a ressonância magnética, o raio- $X$, a tomografia computadorizada e as imagens térmicas. A utilização de câmeras de infravermelho (ou térmicas) na medicina se deu pela evolução dos dispositivos termossensíveis ocorrida nas últimas décadas. A partir do desenvolvimento de câmeras mais precisas, foi renovado o interesse científico pela aplicação desta tecnologia como meio de diagnóstico clínico.

A detecção e acompanhamento adequado da evolução de tumores dependem da qualidade na captura de imagens. Profissionais que realizam a captura de imagens médicas, podendo essas ser mamografias, termografias ou de outra fonte, têm a responsabilidade de realizar a aquisição com alta qualidade diagnóstica. Para tanto, devem ser observadas todas as etapas que envolvem a execução da captura, evitando exposições adicionais da paciente a elementos nocivos (como no exemplo da mamografia ao raio-X) e certificando-se que as imagens capturadas sejam ferramenta úteis para os médicos, uma vez que se forem de qualidade insatisfatória podem resultar em erros de diagnóstico ou exposições desnecessárias.

De acordo com o Instituto Nacional do Câncer $(\text { INCA })^{4}$, as formas mais eficientes para detecção precoce do câncer de mama são o exame clínico da mama e a mamografia, esta última a mais utilizada no Brasil para o diagnóstico precoce da doença. No entanto, em pacientes mais jovens, a mamografia não consegue detectar tumores, devido à maior densidade da mama, uma vez que nessa idade a mama apresenta uma grande quantidade de tecido glandular o que torna esses órgãos densos e firmes. Com o passar da idade esses tecidos vão sendo substituído por tecidos adiposos, menos densos, o que facilita a visualização de patologias. Aliada à idade, a compressão da mama e a radiação ionizante utilizadas na mamografia vêm sendo considerado por alguns pesquisadores um risco para a paciente. Nesse cenário, a termografia mostra-se uma forma auxiliar de diagnóstico precoce do câncer, uma vez que, através da diferença de temperatura, é possível detectar anormalidades em mamas mais densas. Amalu ${ }^{5}$ e $\mathrm{Ng}^{6}$ defendem que termografias anormais persistentes da mama (i.e., sinais térmicos assimétricos que indicam anormalidades fisiológicas que se mantêm inalteráveis) são dez vezes mais significativos do que o histórico familiar da doença no indicativo do desenvolvimento futuro de um tumor cancerígeno.

O registro de imagens, que é o processo de sobreposição de duas ou mais imagens da mesma cena, tomadas em momentos diferentes, a partir de pontos de vista não equivalentes, e/ou por sensores diferentes ${ }^{7}$, é um passo crucial em todas as tarefas de análise de imagem em que o resultado final é obtido pela combinação de várias fontes de dados, em que transformações (ou mapeamentos) devem ser encontradas para que os pontos em uma imagem de referência possam ser relacionados a pontos correspondentes em outras imagens. Portanto, o problema central é determinar a transformação geométrica que alinha imagens aos pares, de tal forma que as diferenças entre as imagens do par sejam minimizadas ou ressaltadas, como no caso da identificação de nódulos em mama cujas imagens foram obtidas antes e após a presença desses nódulos. Tanto Bozek et al ${ }^{8}$ quanto Deshmukh e Bhosle ${ }^{9}$ relatam em suas pesquisas a dificuldade de alinhamento de imagens.

Neste trabalho é apresentado um método automático de verificação de postura de pacientes no momento 
da aquisição de imagens térmicas, objetivando imagens de alta qualidade diagnóstica, o imageamento de todas as regiões da mama que sejam úteis aos diagnósticos, a padronização das capturas e o correto posicionamento de pacientes. A padronização e a garantia de postura resultam em melhorias de extrações automáticas de regiões de interesse (ROI) e no estudo de assimetria térmica para diagnóstico de câncer de mama.

A garantia da postura correta da paciente no momento da captura, faz-se em dois passos:

a. Atualização de protocolos existentes na literatura para que esses capturem todas as regiões do corpo que sejam úteis para o diagnóstico; e

b. Desenvolvimento de um algoritmo para extração de informações da imagem capturada, através do eixo medial, que permitam a verificação de postura da paciente.

\section{Material e Métodos}

Utilizamos neste trabalho um total de 49 termografias adquiridas de acordo com a posição de captura T2. Destas, 32 foram capturadas utilizando protocolo de captura estático e 17, o protocolo de captura dinâmico com ventilação e álcool. Todas possuem 320 pixels de largura e 240 pixels de altura, capturadas utilizando câmeras FLIR, modelos sc620 (41 imagens) e s45 (8 imagens).

\section{Técnica proposta}

A abordagem utilizada tem duas fases. A primeira é uma modificação no protocolo de captura de imagens térmicas, mais especificamente a posição de captura T2. Séries de capturas normalmente utilizadas em alguns grupos de pesquisa do mundo são detalhes no survey de Borchartt et al. ${ }^{10}$. Já na segunda, o objetivo é identificar em uma imagem térmica a porção correspondente ao corpo da paciente sobre a qual se busca encontrar o eixo medial que permitirá, após sua análise, caracterizar como correta ou incorreta a postura da paciente, apontando neste segundo caso, o que estima ser o erro de posicionamento.

\section{Modificação na posição de captura T2}

O novo enquadramento objetiva permitir que a região axilar seja capturada em sua completude, além de permitir um melhor estudo da drenagem dos vasos linfáticos da mama para os gânglios axilares (embaixo dos braços) na região do pescoço e tórax incluímos também, a região do pescoço na imagem a ser capturada na posição T2 padronizada. Este novo enquadramento será utilizado como padrão pelo grupo de pesquisa "VisuaLab" da UFF. Para o novo enquadramento sugerimos que:

a. Se a paciente estiver utilizando óculos, brincos ou colares, estes deverão ser removidos.

b. A paciente deverá enrolar e amarrar os cabelos atrás da cabeça ou utilizar toca descartável.

c. A paciente deverá ficar em posição ereta e corpo verticalmente estendido para cima, então deverá estender os dois braços à frente e entrelaçar os dedos. Logo depois posicionar as mãos entrelaçadas atrás da cabeça de tal forma que os dedos polegares fiquem posicionados nas laterais da cabeça para a obtenção da melhor simetria possível entre os braços.

d. Caso a paciente possua pescoço pequeno ou algum problema nesta região, mover a cabeça para cima de tal forma que esta região se torne visível.

e. O enquadramento correto da imagem para captura deverá ter os seguintes parâmetros: 
- Superior: a boca ou queixo da paciente.

- Inferior: entre 8 e $12 \mathrm{~cm}$ da mama mais baixa de forma a remover possíveis regiões que não sejam de interesse e existentes próximas ao umbigo.

- Esquerda e Direita: o corpo da paciente deverá ficar centralizado no enquadramento da imagem de forma a evidenciar a região mamária e axilar.

\section{Algoritmo de Extração do Eixo Medial}

O processo proposto para verificação da postura da paciente segue um conjunto de passos que vão desde a aquisição de uma imagem até a definição da posição da paciente como correta ou incorreta. Detalhes da implementação são apresentados em Santos $^{3}$ e das técnicas de processamento de imagens que compõem a metodologia proposta são apresentadas em Gonzalez e Woods². Uma visão geral da abordagem proposta é mostrada na Figura 1.

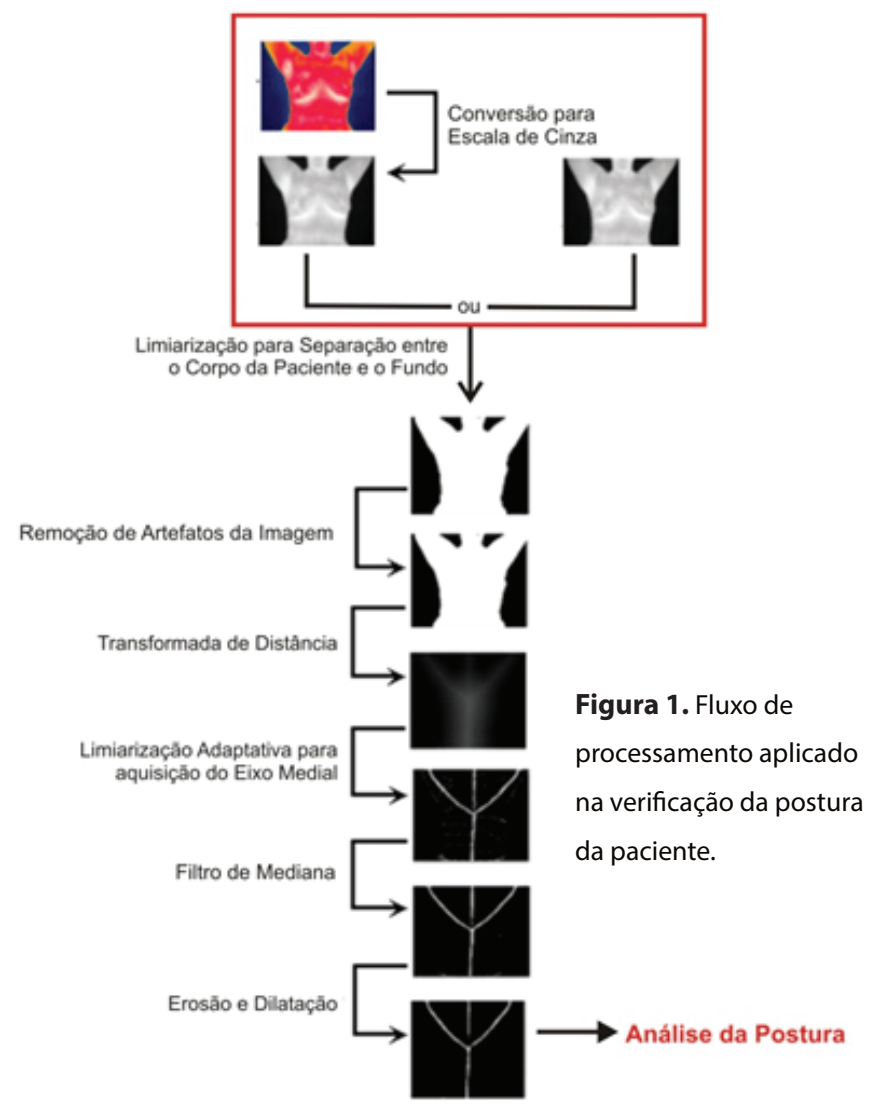

\section{Captura da Imagem}

A imagem térmica antes de ser submetida ao processo, deve ter todas as informações extras removidas, tais como logomarca do fabricante da câmera, ilustrações da paleta no intervalo de temperatura detectadas, dentre outros artefatos.

\section{Conversão para Escala de Cinza}

Logos após a remoção de informações extras a imagem é convertida de RGB para escala de cinza, com valores variando entre 0 (preto) e 255 (branco). Algumas câmeras térmicas permitem que as imagens capturadas sejam transferidas em formato bruto (raw data) via USB, ethernet ou firewire. Neste caso, tanto a fase anterior de processamento quanto esta se tornam desnecessárias; no entanto, ela é mantida para independer do dispositivo de captura.

\section{Limiarização para Separação entre o Corpo da Paciente e o Fundo}

De posse da imagem em escala de cinza é realizada a separação do corpo da paciente do fundo da imagem. Para a tala, é aplicado sobre a imagem em escala de cinza um limiar de valor $\mathrm{T}=50$, obtido empiricamente.

\section{Remoção de Artefatos da Imagem}

Após a aplicação do limiar, em algumas imagens se faz necessário um pré-processamento para remoção de pequenos artefatos provenientes, por exemplo, da presença de brincos, colares e outros ornamentos que não 
foram removidos antes da captura, indo de encontro ao protocolo de aquisição proposto.

\section{Transformada de Distância}

Para a aquisição do eixo medial ou esqueleto do objeto é aplicada uma transformada de distância sobre o corpo da paciente, onde obtém-se o dégradé desta, cujos máximos representam o eixo medial formado pelos pontos internos que se encontram centralizados em relação à borda do objeto e que possuem distância máxima das bordas. A transformada de distância aplicada neste trabalho, utiliza como parâmetros tamanho da máscara 5 X 5 e métrica Euclidiana.

\section{Limiar Adaptativo para Extração do Eixo Medial}

O dégradé obtido na etapa anterior mostra-se, ainda, não muito visível. Objetivando tornar o eixo medial destacado em relação ao restante da imagem, aplicamos um limiar adaptativo para realçar a estrutura esquelética em forma de "tridente" que representa o eixo medial da imagem. O limiar adaptativo aplicado neste trabalho foi o mean-c, com janela de tamanho $9 \times 9$ e constante $\mathrm{c}=0$.

\section{Filtro de Mediana}

Com a aplicação do limiar adaptativo o tridente esperado torna-se aparente. Porém, uma grande quantidade de artefatos (tradicional ruído sal-epimenta) aparece na imagem resultante. Para a remoção destes ruídos da imagem é utilizado um filtro da mediana $3 \times 3 . b$

\section{Erosão e Dilatação}

A utilização do filtro de mediana mostrou-se bastante efetiva na remoção dos ruídos da imagem. No entanto, observou-se nos experimentos que alguns artefatos permanecem após sua aplicação. Para a remoção dos ruídos remanescentes aplica-se um novo processo de erosão seguido por dilatação.

\section{Análise do Eixo Medial}

De posse do eixo medial, a análise é realizada em duas etapas. A primeira visa encontrar os pontos que servirão de base para a análise do eixo medial: um ponto inferior, três superiores e um ponto central ou de junção. A localização dos pontos base encontra-se ilustrada na Figura 2.

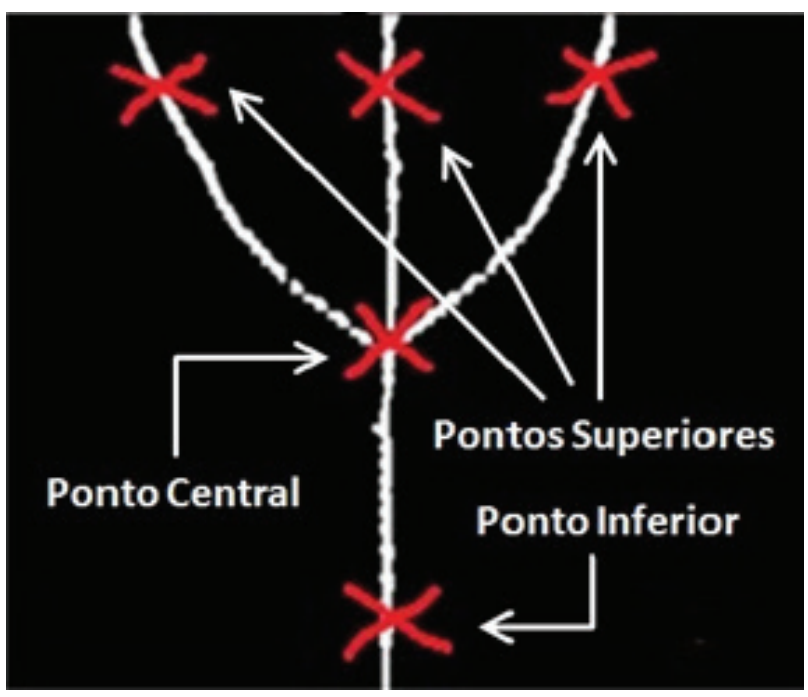

Figura 2. Localização dos pontos de análise do eixo medial, sendo um inferior, três superiores e um central

A segunda etapa da análise é realizada através da verificação de semelhança (i.e., simetria) entre as regiões localizadas à esquerda e à direita da imagem divididas pelo segmento do tronco da paciente e localizadas abaixo do ponto de junção. 


\section{Análise da postura da paciente}

Uma vez localizados os pontos que representam a localização dos segmentos superiores $(A, B, C)$, central (D) e inferior (E) traçamos uma estrutura semelhante a um tridente composta de quatro segmentos de reta $A B, B D, E D$ e $C D$, tendo $D$ como ponto comum. A estrutura formada por estes pontos encontra-se ilustrada na Figura 3.

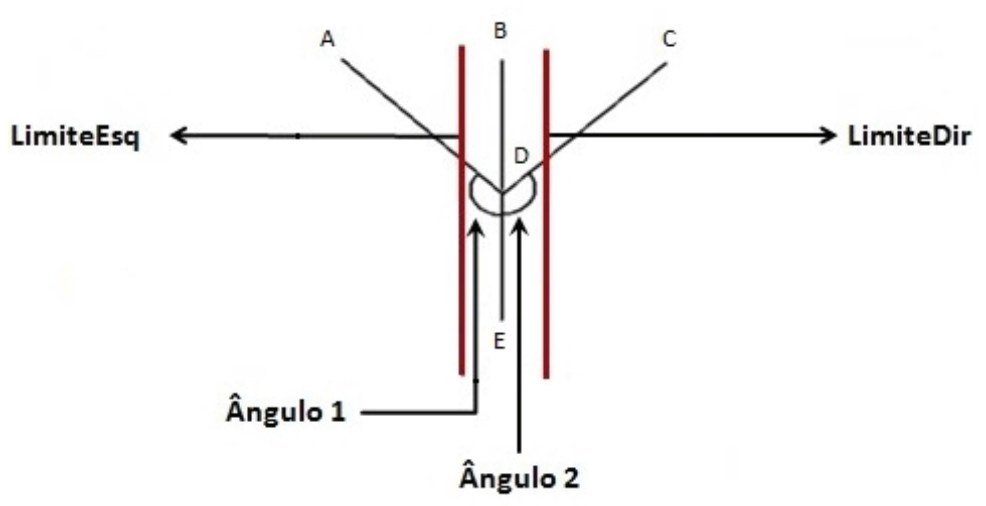

Figura 3. Segmentos de reta $A D, B D, C D$ e $D E$ no domínio contínuo, formados pela ligação dos pontos base. A região limite para os pontos B e E é representada por uma linha em vermelho a esquerda e outra a direita do ponto D. Esta imagem ilustra a localização dos ângulos 1 e 2.

O processo de verificação de postura proposto utiliza três condições diferentes, porém complementares, para detectar casos desfavoráveis de postura da paciente. A primeira é derivada da análise horizontal dos pontos $B$ e $E$ em relação a $D$, sendo que esses deverão estar localizados dentro de uma região preestabelecida determinada em função de um percentual de $2 \%$ dos pixels (largura da imagem) à esquerda e à direita de D. A segunda condição utiliza a comparação entre os ângulos formados pelos segmentos de reta $\mathrm{ADE}$ (ângulo 1) e CDE (ângulo 2) para detectar casos de flexão lateral, esperando que os ângulos sejam aproximadamente iguais, aceitando uma diferença mínima de $8^{\circ}$ entre eles. $E$ a terceira condição utiliza a verificação de simetria com o propósito detectar casos de torção, ilustrado na Figura 4.

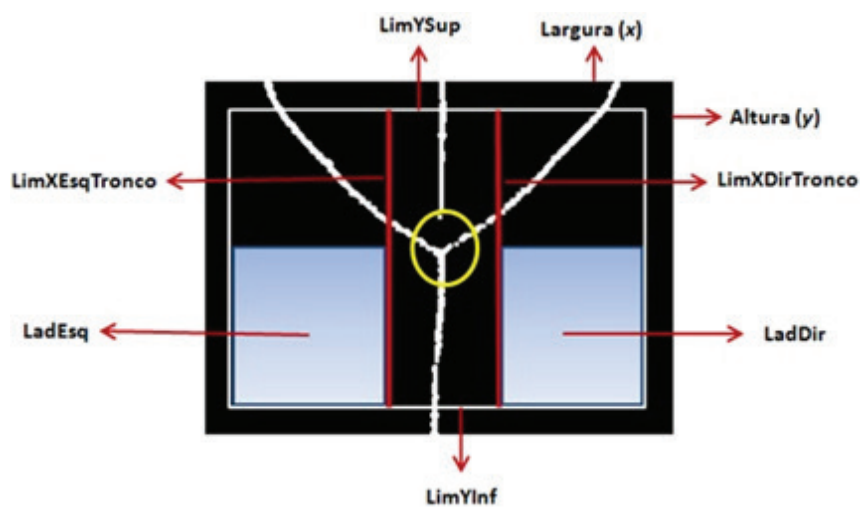

Figura 4. Áreas de simetria, indicadas pelos retângulos em tons de azul, localizadas ao lado esquerdo (LadEsq) e ao lado direito (LadDir) da extremidade inferior do eixo medial.

As combinações testadas pela técnica proposta encontram-se enumeradas na Tabela I, composta por quatro colunas, sendo as três primeiras: pontos, ângulos e simetria que podem receber o valor verdadeiro (condição validada) ou falso (condição não validada). Na primeira coluna é ilustrada a condição dos pontos $B$ e $E$ em relação a $D$. Já na segunda coluna é observada a condição de diferença entre os ângulos, enquanto que na terceira coluna encontramos a condição de simetria. A quarta coluna, denominada "situação", é baseada nos valores verdadeiro ou falso das colunas anteriores onde: (i) se todas as colunas possuírem valor Verdadeiro determinar-se-á a postura correta, (ii) caso uma destas colunas possua valor Falso determinarse-á a postura incorreta por torção ou flexão.

\begin{tabular}{|l|l|l|l|}
\hline Pontos & Ângulos & Simetria & Situação \\
\hline Verdadeiro & Verdadeiro & Verdadeiro & Postura correta \\
\hline Verdadeiro & Verdadeiro & Falso & $\begin{array}{l}\text { Problema de } \\
\text { Torção }\end{array}$ \\
\hline Verdadeiro & Falso & Falso & $\begin{array}{l}\text { Problema de } \\
\text { Torção }\end{array}$ \\
\hline Falso & Falso & Verdadeiro & $\begin{array}{l}\text { Problema de } \\
\text { Flexão Lateral }\end{array}$ \\
\hline Falso & Verdadeiro & Verdadeiro & $\begin{array}{l}\text { Problema de } \\
\text { Flexão Lateral }\end{array}$ \\
\hline Falso & Verdadeiro & Falso & $\begin{array}{l}\text { Problema de } \\
\text { Flexão Lateral }\end{array}$ \\
\hline Falso & Falso & Falso & $\begin{array}{l}\text { Problema de } \\
\text { Flexão Lateral }\end{array}$ \\
\hline
\end{tabular}

Tabela1. Combinação das condições de postura. 


\section{Conclusão}

Neste trabalho apresentamos uma técnica de verificação da postura de pacientes no processo de captura de imagens térmicas com o objetivo de auxiliar métodos de extração automática de ROI e métodos de diagnóstico assistido por computador (CAD) na realização de suas tarefas. É importante frisar que a utilização do eixo medial como elemento fundamental na verificação do posicionamento da paciente é o fato de, apesar de ser calculado em função silhueta, o comportamento esperado para o eixo na posição de captura T2 é pouco suscetível a alterações em função das características gerais da silhueta. Uma consequência direta deste fato é a robustez da técnica proposta quando esta é aplicada sobre imagens de pacientes altas, baixas, gordas ou magras.

Apesar de a técnica descrita neste trabalho ser direcionada a imagens térmicas, mais especificamente à posição de captura $T 2$, sua adaptação para outros tipos de exames que requeiram posicionamento e posições de captura pode ser feita através de modificações na forma de análise do eixo medial, uma vez que todas possuem características particulares que geram estruturas de eixos mediais também diferentes.

\section{Agradecimentos}

Agradecemos à CAPES, pois algumas imagens utilizadas no desenvolvimento deste trabalho foram capturadas com equipamentos adquiridos com o apoio desta, através dos projetos Pro-Engenharias no 021/2007 e Pro CAD-NF no 08/2008. À pró-reitoria de pesquisa e inovação do IFPI (PROPI) pela bolsa concedida para a realização desta pesquisa.

\section{Referências}

1. R. S. Marques, "Segmentação automática das mamas em imagens térmicas," Master's thesis, Universidade Federal Fluminense, 2012

2. GONZALEZ, R.C., WOODS, R.E. Processamento de imagens digitais, Editora Edgard Blücher, São Paulo, 2000

3. D. A. Santos, "Uma técnica baseada em imagens para correção da postura de pacientes na aquisição de termografias," Master's Thesis, Programa de Pós-Graduação em Computação, Universidade Federal Fluminense, Niterói, RJ, 2012.

4. INCA. Portal do Instituto Nacional do Câncer. Disponível em: \&lt;http://www2.inca. gov.br\&gt; ${ }_{\text {i }}$ Acessado em 10 de fevereiro de 2014.

5. AMALU, W. C. A Review of Breast Thermography. Redwood City (EUA): International Academy of Clinical Thermography (IACT), 2002.

6. NG, E.Y.K. A review of thermography as promising non-invasive detection modality for breast tumor, In: International Journal of Thermal Sciences, vol. 48, p. 849-859, 2008.

7. ZITOVA, B., FLUSSER, J. Image registration methods: a survey. Image and Vision Computing, vol. 21, p. 1-36, 2003

8. BOZEK, J., MUSTRA, M.,GRGIV, M. A survey of mammographic image processing algorithms for bilateral asymmetry detection, In: $51^{\circ}$ International Symposium ELMAR, p. 9-14, 2009.

9. DESHMUKH, M., BHOSLE, U. A survey of image registration. International Journal of image processing, vol. 5, p. 245-269, 2011.

10. BORCHART, T.B., CONCI, A., LIMA, R.C.F., RESMINI, R., SANCHES, A. Breast thermography from an image processing viewpoint: a survey. Signal Processing (2013). 